\title{
Estimands in diabetes clinical trials
}

Thinzar Min ${ }^{1,3}$, Senior Clinical Lecturer in Diabetes \& Endocrinology

Stephen C Bain ${ }^{2,3}$, Professor of Medicine (Diabetes)

Neath Port Talbot Hospital ${ }^{1}$ \& Singleton Hospital ${ }^{2}$, Swansea Bay University Health Board and Swansea University Medical School ${ }^{3}$, Swansea UK.

During 2019 several trials in the field of type 2 diabetes have been published, which report results using the term 'estimand'. This will be an unfamiliar word to many clinicians (and to spellcheck) but given that regulatory bodies have endorsed its use, it is likely to become a staple of medical jargon in the future.

\section{Introduction}

Randomised controlled trials (RCT) have been recognised as the gold standard to quantify the treatment effect of a new medication because randomisation reduces confounding factors and biases at baseline. However, during clinical trials it is inevitable that events occur after the initiation of investigational medical product (IMP), which affect the interpretation of the trial results (usually termed 'intercurrent events')[1]. Intention-to-treat (ITT) analyses have been widely employed in clinical trials to assess the clinical effectiveness of the treatment under investigation. The ITT principle requires that all randomised participants are included in the final analysis, according to the treatment group to which they were originally assigned and regardless of the occurrence of intercurrent events[2].

Intercurrent events may present in many forms, one of the most common in diabetes trials being the use of rescue medication[3]. For ethical reasons, patients can receive additional glucose lowering medication if they fail to achieve the glucose targets specified in the protocol. If subjects receive rescue medication, then glucose-lowering may be achieved but will reflect the combined effect of the IMP plus the rescue medication. Other intercurrent events include premature discontinuation of IMP, loss to follow-up and death. When a patient discontinues IMP because of adverse events, there will be a final glucose outcome, but this will not be due to the trial medication. In contrast, for patients who are lost to follow-up or deceased, there will be no observed value at the end of the study. In the past, the last observed value before the use of rescue medication, discontinuation of IMP or loss to follow-up was analysed ('carried forward') as the end-of-trial value. This is known as the 'last- 
observation-carried-forward' (LOCF) approach and was endorsed by the Food and Drug Administration (FDA) in its 2008 guidance[4]. The LOCF approach, however, can overestimate the effect of a trial product because it assumes that patients who have a good short-term outcome will continue to have a good outcome going forwards[5]. Conversely, LOCF will underestimate an effect that only slowly appears if a trial subject drops out early from the study. The 2010 National Research Council Report, commissioned by the FDA, acknowledged these deficits and recommended against the use of LOCF[6]; this advice was also endorsed by the European Medicines Agency[5].

The FDA's former position on the LOCF approach was illustrated in the 2011 public advisory committee meeting for dapagliflozin[3]. The trial sponsor excluded data accrued after the initiation of rescue medication, aiming to establish the true treatment effect of dapagliflozin. However, the FDA reviewer argued that all data should be included regardless of the use of rescue medication. This clearly demonstrated that the scientific question of interest can differ according to different stakeholders' perspectives. Trial protocols or publications have previously not completely described the type of treatment effect to be estimated, and this has led to difficulty in interpreting data and making comparisons of results from other trials. In 2017, the International Council for Harmonisation (ICH) Steering Committee published a draft guideline entitled 'Estimands and sensitivity analysis of clinical trials' (ICH E9 (R1)) and this is now being adopted.

\section{The estimand concept}

An estimand is "a detailed description of what needs to be estimated to address a specific scientific question of interest" [1] and is characterised according to four elements: (a) the population of interest; (b) the endpoint of interest; (c) the methods for handling intercurrent events; and (d) the population level summary. Traditionally, trial protocols have not explicitly described the third element and the ICH E9 (R1) draft paper proposed five different strategies to deal with this:

a. Treatment policy strategy in which the observed values of a variable are used regardless of intercurrent events. This broadly corresponds to the previously recommended ITT analysis.

b. Hypothetical strategy in which a situation is envisaged where all participants adhered to the protocol and do not experience any intercurrent events.

c. Composite strategy where the intercurrent event is integrated with one or more measures of clinical outcome as a combined variable of interest.

d. Principal stratum strategy where only the subset of the trial population who did not experience the intercurrent event is subjected to the analysis. 
e. While on treatment strategy which describes the treatment effect before any intercurrent events occurred. In this strategy, the outcomes until the time of the intercurrent event are analysed, rather than the value observed at the same fixed timepoints for all participants.

It should be noted that none of these analyses directly correspond to a per-protocol or complete case analysis.

In 2018 the EMA published draft guidance discussing the specific use of estimands in diabetes trials [7]. When investigating the change from baseline HbA1c, the EMA recommends the hypothetical strategy' for the use of rescue medication; the 'treatment policy strategy' for premature discontinuation of IMP; and the 'composite strategy' to account for two intercurrent events through definition of the endpoint. Following these recommendations from the FDA and the EMA, several diabetes clinical trials have incorporated the estimand concept including three in Lancet Diabetes \& Endocrinology [8-10].

To put these estimands into the context of a diabetes trial (see figure), the hypothetical estimand (also termed 'trial product estimand') will tell the clinician the glucose-lowering effect of a new treatment if the patient is willing and able to take it as directed, whilst the treatment policy estimand will indicate how the drug will work in a more real-life setting where drug discontinuation and add-in therapies are common (a scenario more relevant to payers). The composite estimand may seek to combine these facets, for example patients reaching glycaemic targets whilst adhering to trial product and not taking additional therapy.

An obvious question is how much the trial endpoints are affected when different estimands are considered and data are now available. For example, in the PIONEER 5 trial of oral semaglutide versus placebo, the mean $\mathrm{HbA1c}$ and weight changes from baseline to 26 weeks were $-1.1 \%(-12 \mathrm{mmol} / \mathrm{mol})$ and $-3.7 \mathrm{~kg}$ for the hypothetical estimand compared with $-1.0 \%(-11 \mathrm{mmol} / \mathrm{mol})$ and $-3.4 \mathrm{~kg}$ using the treatment policy estimand [9]. So, there are the anticipated differences in favour of the hypothetical estimand but the magnitude is small for both $\mathrm{HbA1c}$ and weight.

1. ICH. International Council for Harmonisation (ICH) of Technical Requirements for Pharmaceuticals for Human Use. ICH Harmonised Guideline E9 (R1): Estimands and Sensitivity Analysis in Clinial Trials. 2017 30/09/2019].

2. Fergusson, D., et al., Post-randomisation exclusions: the intention to treat principle and excluding patients from analysis. BMJ, 2002. 325(7365): p. 652-4.

3. Holzhauer, B., M. Akacha, and G. Bermann, Choice of estimand and analysis methods in diabetes trials with rescue medication. Pharm Stat, 2015. 14(6): p. 433-47. 
4. FDA. Guidance for Industry. Diabetes Mellitus: Developing Drugs and Therapeutic Biologics for Treatment and Prevention. 2008 03/10/2019].

5. $\quad$ EMA. Guideline on Missing Data in Confirmatory Clinical Trials. 2010 4/10/19].

6. NRC, National Research Council (US) Panel on Handling Missing Data in Clinical Trials. The Prevention and Treatment of Missing Data in Clinical Trials. 2010.

7. EMA. Guideline on clinical investigation of medicinal products in the treatment or prevention of diabetes mellitus. 2018 04/10/2019].

8. Pieber TR, Bode B, Mertens A, Cho YM, Christiansen E, Hertz CL, Wallenstein SOR, Buse JB; PIONEER 7 investigators.Efficacy and safety of oral semaglutide with flexible dose adjustment versus sitagliptin in type 2 diabetes (PIONEER 7): a multicentre, open-label, randomised, phase 3a trial. Lancet Diabetes Endocrinol. 2019 Jul;7(7):528-539. doi: 10.1016/S2213-8587(19)30194-9.

9. Mosenzon, O., et al., Efficacy and safety of oral semaglutide in patients with type 2 diabetes and moderate renal impairment (PIONEER 5): a placebo-controlled, randomised, phase $3 a$ trial. Lancet Diabetes Endocrinol, 2019. 7(7): p. 515-527.

10. Lingvay I, Catarig AM, Frias JP, Kumar H, Lausvig NL, le Roux CW, Thielke D, Viljoen A, McCrimmon RJ. Efficacy and safety of once-weekly semaglutide versus daily canagliflozin as add-on to metformin in patients with type 2 diabetes (SUSTAIN 8): a double-blind, phase 3b, randomised controlled trial. Lancet Diabetes Endocrinol. 2019 Nov;7(11):834-844. doi: 10.1016/S2213-8587(19)30311-0. 